\title{
CD80 and CD86 knockdown in dendritic cells regulates Th1/Th2 cytokine production in asthmatic mice
}

\author{
JIAN-GUO LI $^{1 *}$, YU-MO DU $^{1 *}$, ZHI-DONG YAN ${ }^{1}$, JIA YAN $^{2}$, YONG-XUN ZHUANSUN $^{1}$, \\ RUI CHEN ${ }^{1}$, WEI ZHANG ${ }^{1}$, SU-LING FENG ${ }^{1}$ and PI-XIN RAN ${ }^{3}$ \\ ${ }^{1}$ Department of Respiratory Medicine, Sun Yat-Sen Memorial Hospital, Institute of Respiratory Diseases, \\ Sun Yat-Sen University, Guangzhou, Guangdong 510120; ${ }^{2}$ Department of Intensive Care Unit, \\ Tianjin Medical University General Hospital, Tianjin 300052; ${ }^{3}$ Department of Respiratory Medicine, \\ The First Affiliated Hospital of Guangzhou Medical College, Guangzhou, Guangdong 510120, P.R. China
}

Received November 6, 2014; Accepted December 14, 2015

DOI: 10.3892/etm.2016.2989

\begin{abstract}
Dendritic cells (DCs) are associated with the activation and differentiation of $\mathrm{T}$ helper (Th) cells. Cluster of differentiation (CD)80 and CD86, the co-stimulatory molecules highly expressed in DCs, have are prominent in promoting the differentiation of Th cells toward Th2 cells. However, little is known about the effect of CD80 and CD86 knockdown on Th1/Th2 cytokine production in mature DCs (mDCs). The aim of the present study was to investigate whether small-interfering RNA (siRNA) could suppress the surface expression of CD80 and CD86 in mDCs. The effects of CD80 and CD86 knockdown in mDCs on Th1/Th2 cytokine expression were examined using an asthmatic murine model. DCs were isolated, separated and cultured in vitro. Flow cytometry was used to examine the expression of CD11c, CD80 and CD86 on the DCs. The DCs were transfected with CD80- and CD86-specific siRNA, while non-siRNA and negative siRNA controls were also designed. Then, the mRNA and protein expression levels of CD80 and CD86 were determined by reverse transcription-quantitative polymerase chain reaction and flow cytometry, respectively. The levels of interferon (IFN)- $\gamma$ and interleukin (IL)- 4 produced by $T$ cells co-cultured with mDCs were measured by enzyme-linked immunosorbent assay. Substantial downregulation of CD80 and CD86 mRNA and protein levels were observed in the mDCs following transfection with siRNA. The level of IFN- $\gamma$ produced by $\mathrm{T}$ cells co-cultured with mDCs was significantly increased
\end{abstract}

Correspondence to: Professor Jian-Guo Li, Department of Respiratory Medicine, Sun Yat-Sen Memorial Hospital, Institute of Respiratory Disease, Sun Yat-Sen University, 107 Yanjiang West Road, Guangzhou, Guangdong 510120, P.R. China

E-mail: jgli88@126.com

*Contributed equally

Key words: small-interfering RNA, dendritic cell, CD80, CD86, Th1/Th2, asthma in the siRNA group, while IL-4 production was significantly decreased. These results show that specific targeting of CD80 and CD86 with siRNA is able to suppress CD80/CD86 expression and consequently regulate Th1/Th2 cytokine levels by increasing IFN- $\gamma$ production and decreasing IL-4 levels in an asthmatic murine model.

\section{Introduction}

Bronchial asthma is a chronic inflammatory airway disease involving many inflammatory cells and mediators. T cells, particularly $\mathrm{T}$ helper (Th)1 and Th2. have a crucial role in the induction of airway inflammation in asthmatic patients (1). Complicated immune responses are capable of inducing Th1 deficiency and Th2 hyperactivity, which results in a Th1/Th2 imbalance. This imbalance promotes immunoglobulin (Ig)E secretion and sensitizes mastocyte and eosinophils via altered cytokine secretion and causes allergic inflammation and hyper-responsiveness of the airway $(2,3)$. Interferon (IFN)- $\gamma$ and interleukin (IL)-4 are typical cytokines of Th1 and Th2, respectively.

In patients with asthma, persistent airway inflammation is initiated by antigen presenting cells (APC), which integrate various allergens into a signal for $\mathrm{T}$ cells and prime the subsequent immune responses $(4,5)$. Activation of $\mathrm{T}$ cells requires signals which are initiated via the TCR complex and cluster of differentiation (CD)28. Mature dendritic cells (mDCs) express high levels of the co-stimulatory molecules CD80 and CD86, which provide the signal that is required for triggering $\mathrm{T}$ cell activation, expansion and differentiation via interaction with CD28 (6). Previous studies have demonstrated that CD80 and CD86 levels are elevated in patients with asthma $(7,8)$.

In previous studies investigating asthmatic models, mDCs have been shown to induce Th2 polarization, upregulate IL-4 secretion, downregulate IFN- $\gamma$ production and induce eosinophilic inflammation $(9,10)$. However, studies investigating the effects of the knockdown of CD80 and CD86 in DCs on the differentiation of and cytokine secretion by $\mathrm{T}$ helper cells in murine models of asthma are lacking.

In the present study, co-stimulatory T-cell activation signals were blocked by the suppression of CD80 and CD86 molecule 
expression on DCs using small interfering RNA (siRNA), and the effects of CD80 and CD86 knockdown on the expression levels of the Th1/Th2 typical cytokines IFN- $\gamma$ and IL-4 were evaluated. Thus, the potential of CD80 and CD86 as targets for the application of RNA interference (RNAi) in the therapeutic targeting of asthma were investigated.

\section{Materials and methods}

Animals. A total of 20 healthy specific-pathogen-free grade BALB/c mice (6-8 weeks; mean weight, $18 \pm 2 \mathrm{~g}$ ) were purchased from the Center of Experimental Animals of Sun Yat-Sen University (Guangzhou, China). Experiments were performed according to protocols approved by the Animal Studies Committee of Sun Yat-Sen University.

Asthma models. A total of 20 mice were randomly assigned to two groups: i) the asthmatic group, and ii) the normal control group. In the asthmatic group, each mouse was sensitized to ovalbumin (OVA; Sigma-Aldrich, St. Louis, MO, USA) intraperitoneally on days 1,14 and 21 with $100 \mu \mathrm{g}$ OVA that was emulsified in $20 \mathrm{mg}$ alum (Guangzhou Chemical Reagent Co., Guangzhou, China). Subsequently, the mice were exposed to an aerosol challenge of 5\% OVA for $30 \mathrm{~min} /$ day in airtight containers (with dimensions of $50 \times 50 \times 50 \mathrm{~cm}$ ) on days 28-34. In the normal control group, mice were sensitized and challenged as above with an equivalent amount of saline solution instead of the OVA protein solution. At $24 \mathrm{~h}$ after the last challenge, mice were sacrificed by an approved cervical dislocation procedure conducted by skilled and fully trained personnel. The lungs were removed and then fixed in $10 \%$ ethanol for $24 \mathrm{~h}$. Specimens were dehydrated, embedded in paraffin, and stained with hematoxylin and eosin as previously described (11). Pathological changes in bronchial and lung tissues were assessed under a Nikon Eclipse Ti light microscope (Nikon Corporation, Tokyo, Japan).

Separation of bone marrow-derived DCs. All mice were sacrificed by cervical dislocation $24 \mathrm{~h}$ after the final challenge. Bone marrow was flushed from the femurs and tibiae with RPMI-1640 culture medium (Gibco; Thermo Fisher Scientific, Inc., Waltham, MA, USA). Following centrifugation at $250 \mathrm{x} \mathrm{g}$ for 5 min, cells were treated with red blood cell (RBC) lysis buffer (CWBio Co., Ltd., Beijing, China), washed with phosphate-buffered saline (PBS), centrifuged at $250 \mathrm{x}$ g for $5 \mathrm{~min}$ and cultured in RPMI-1640 supplemented with recombinant mouse granulocyte macrophage colony-stimulating factor (rmGM-CSF; Peprotech, Inc., Rocky Hill, NJ, USA; 10 ng/ml), and rmIL-4 (Peprotech; $10 \mathrm{ng} / \mathrm{ml}$ ) were used in turn. After 6 days of culture, $1 \mu \mathrm{g} / \mathrm{ml}$ lipopolysaccharide (LPS; Sigma-Aldrich) was added, and the non-adherent $\mathrm{mDCs}$ were harvested on day 7. DCs were stained at $4^{\circ} \mathrm{C}$ for $30 \mathrm{~min}$ with fluorescein isothiocyanate (FITC)-conjugated hamster anti-CD11c (5 $\mu \mathrm{g} / \mathrm{ml} ; 11-0114)$, phycoerythrin (PE)- conjugated hamster anti-CD80 $(5 \mu \mathrm{g} / \mathrm{ml}$; 12-0801), FITC-conjugated rat anti-CD86 (5 $\mu \mathrm{g} / \mathrm{ml} ; 11-0862)$ and PE-conjugated rat anti-major histocompatibility complex (MHC) II ( $5 \mu \mathrm{g} / \mathrm{ml}$; 12-5322; all eBioscience,Inc.,San Diego,CA, USA) monoclonal antibodies, and were subsequently analyzed by flow cytometry (BD FACSVerse; BD Biosciences, Franklin Lakes, NJ, USA) in order to determine the positive expression rate of the labeled antigen expression.
siRNA and transfection. The specific siRNA sequences (Table I) targeting CD80 and CD86 were designed and selected according to the methods of Gu et al (12). All siRNA was purchased from Shanghai GenePharma Co., Ltd. (Shanghai, China). The transfection step was performed according to the manufacturer's protocol for Lipofectamine 2000 (Invitrogen; Thermo Fisher Scientific, Inc.). Briefly, DCs were cultured in a 24-well tissue culture plate at a density of $1 \times 10^{5}$ cells/well on the day prior to transfection. To prepare lipid-siRNA complexes, $3 \mu \mathrm{l}$ Lipofectamine 2000 was incubated in $50 \mu \mathrm{l}$ Opti-MEM (Gibco; Thermo Fisher Scientific, Inc.) at room temperature for $5 \mathrm{~min}$, and $12 \mu \mathrm{l}$ of the indicated siRNA was concurrently combined with $50 \mu$ l Opti-MEM. The diluted Lipofectamine 2000 and siRNA were subsequently mixed and incubated for a further $20 \mathrm{~min}$ at room temperature for complex formation. Subsequently, the complex was incubated with the DCs in a 24-well plate at $37^{\circ} \mathrm{C}$ in a $5 \%$ humidified $\mathrm{CO}_{2}$ in air atmosphere for $6 \mathrm{~h}$. When cotransfection was performed, equivalent amounts of CD80 siRNA:Lipofectamine 2000 and CD86 siRNA:Lipofectamine 2000 complexes were added to each well. FAM-scrambled-siRNA was used as the negative control in order to determined the transfection efficiency. Three groups of transfected DCs were established: In the non-siRNA group, only Lipofectamine 2000 was added, without any siRNA being added to the DCs. In the siRNA group, mDCs were cotransfected by CD80- and CD86-specific siRNA. In the negative siRNA group, $\mathrm{mDC}$ were transfected by non-specific non-targeting FAM-siRNA, which has no homology with the targeted RNAs. Experiments were performed in triplicate for each sample. Transfection efficiency was determined using fluorescence microscopy (Nikon Eclipse Ti, Nikon Corporation) and detected by flow cytometry.

Reverse transcription-quantitative polymerase chain reaction (RT-qPCR). To evaluate CD80 and CD86 mRNA expression levels following transfection, RT-qPCR was performed. Primer sequences (Table II) were designed according to GenBank and synthesized by DaAn Gene Co., Ltd. of Sun Yat-Sen University (Guangzhou, China). At $24 \mathrm{~h}$ post-transfection, the total RNA of $1 \times 10^{6}$ DCs was extracted using TRIzol reagent (Invitrogen; Thermo Fisher Scientific, Inc.) and reverse transcribed and amplified using QuantiTect SYBR Green RT-PCR kit (Qiagen $\mathrm{GmbH}$, Hilden, Germany) in a Roche LightCycler 480 (Roche Diagnostics, Basel, Switzerland). The amplifications were carried out according to the manufacturer's protocol for the QuantiTect SYBR Green RT-PCR kit (Takala, Japan). Amplification conditions were 40 cycles of $93^{\circ} \mathrm{C}$ for $3 \mathrm{~min}$, $93^{\circ} \mathrm{C}$ for $30 \mathrm{sec}, 55^{\circ} \mathrm{C}$ for $45 \mathrm{sec}$ and $72^{\circ} \mathrm{C}$ for $45 \mathrm{sec}$. Every sample was administered to each of three wells. Relative gene expression levels were calculated using the quantification cycle (Cq) method with normalization to glyceraldehyde-3-phosphate dehydrogenase (GAPDH) as the reference gene using $2^{-\Delta \Delta \mathrm{Cq}}(13)$.

Flow cytometry. To detect the positive expression rates of CD80 and CD86 on the DCs following transfection, flow cytometry was performed on the MHC II/CD11c gate for CD80 and CD86. Six hours after transfection, DCs were washed twice with PBS and incubated with fluorescently-labeled antibody at $4^{\circ} \mathrm{C}$ for $30 \mathrm{~min}$. Subsequently, the cells were washed again with PBS and fixed with $10 \mathrm{~g} / \mathrm{l}$ paraformaldehyde. The following 
Table I. Sequences of siRNA.

\begin{tabular}{lll}
\hline siRNA & \multicolumn{1}{c}{ Sense $\left(5^{\prime} \rightarrow 3^{\prime}\right)$} & \multicolumn{1}{c}{ Antisense $\left(5^{\prime} \rightarrow 3^{\prime}\right)$} \\
\hline CD80 siRNA & GGAAAGAGGAACGUAUGAAdTdT & UUCAUACGUUCCUCUUUCCdTdT \\
CD86 siRNA & CAGAGAAACUUGAUAGUGUdTd & ACACUAUCAAGUUUCUCUGdTd \\
FAM siRNA & UUCUCCGAACGUGUCACGUTT & ACGUGACACGUUCGGAGAATT
\end{tabular}

siRNA, small interfering RNA; CD, cluster of differentiation.

Table II. Primer sequences of mRNA.

\begin{tabular}{lll}
\hline mRNA & \multicolumn{1}{c}{ Forward $\left(5^{\prime} \rightarrow 3^{\prime}\right)$} & \multicolumn{1}{c}{ Reverse $\left(5^{\prime} \rightarrow 3^{\prime}\right)$} \\
\hline CD80 & CTGGGAAAAACCCCCAGAAG & TGACAACGATGACGACGACTG \\
CD86 & CATGGGCTTGGCAATCCTTA & AAATGGGCACGGCAGATATG \\
GAPDH & CGTGTTCCTACCCCCAATGT & TGTCATCATACTTGGCAGGTTCT \\
\hline
\end{tabular}

GAPDH, glyceraldehyde 3-phosphate dehydrogenase; CD, cluster of differentiation.

anti-mouse monoclonal antibodies were used: PE-anti-MHC II, FITC-anti-CD11c, PE-anti-CD80, and FITC-anti-CD86, as mentioned above. All flow cytometric analyses were performed using $\operatorname{IgG}$ isotypic controls.

T-cell separation. Spleens were removed after the mice had been euthanized by cervical dislocation. T cells were separated using Mouse Lymphocyte Separation Medium according to the manufacturer's protocol (Dakewe Biotech Co., Ltd., China). The cell density was adjusted to $1 \times 10^{9} / 1$ prior to further processing.

Mixed lymphocyte reaction $(M L R)$. The asthmatic murine

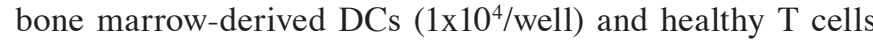
(1x10 $/$ well) were co-cultured in 96-well plates at a 1:10 ratio. The co-culture systems were divided into three groups: i) The non-siRNA group, ii) the siRNA group, and iii) the negative siRNA group, with DCs from the corresponding groups as described above. Next, OVA was added to each well to a final concentration of $10 \mathrm{mg} / \mathrm{l}$ in a total volume of $200 \mu \mathrm{l}$. The cells were incubated at $37^{\circ} \mathrm{C}$ in a $5 \%$ humidified $\mathrm{CO}_{2}$ in air atmosphere for $72 \mathrm{~h}$.

Enzyme-linked immunosorbent assays (ELISAs). After 3 days of co-culture, the supernatant was collected. IFN- $\gamma$ and IL-4 levels were analyzed using ELISA kits specific for IFN- $\gamma$ and IL-4 (Dakewe Biotech Co., Ltd.) according to the manufacturer's instructions. Absorbance values were read at $450 \mathrm{~nm}$ using a Multiskan MK3 (Thermo Fisher Scientific, Inc.).

Statistical analysis. Statistical analyses were performed with SPSS software, version 13.0 (SPSS, Inc., Chicago, IL, USA). Data are presented as the mean \pm standard deviation (SD). Examinations were carried out in triplicate for each mouse. Statistical comparisons between groups were performed using one-way analysis of variance, and comparisons within a group were performed using Student's t-test. Differences among groups were considered statistically significant when $\mathrm{P}<0.05$.

\section{Results}

Asthmatic model. The 20 healthy SPF-grade BALB/c mice were assigned to asthmatic and normal control groups, with 10 mice in each. All mice were evaluated in the final analysis without experimental animal loss. According to the lung tissue pathology, lung sections from the OVA-immunized mice showed clear airway inflammation with peribronchiolar and perivascular infiltrates. These infiltrates consisted predominantly of eosinophils and lymphocytes, and sections showed bronchial mucosa and smooth muscle thickening, increased mucus secretion, airway epithelial cell shedding, airway stenosis, and inflammatory cells scattered in the lung interstitium. No significant pathological changes were observed in lung sections from the normal control group. Representative histopathological data are shown in Fig. 1.

Cell surface molecule expression by $m D C s$. The expression levels of CD11c, CD80 and CD86 on the mDC surfaces were detected by fluorescence-activated cell sorting (FACS). mDCs from the asthmatic group expressed CD11c at a level comparable with that in the normal control group; no significant difference was found between the two groups $(\mathrm{P}>0.05)$. However, in comparison with the normal control group, the asthmatic group showed significantly higher CD80 and CD86 expression levels ( $\mathrm{P}<0.05$; Fig. 2).

Transfection of mDCs. The CD80- and CD86-specific siRNA constructs were successfully transferred into the mDCs. The transfected mDCs were observed under a fluorescence microscope $6 \mathrm{~h}$ after transfection. The transfection efficiency of siRNA detected by FACS was $\sim 75 \%$ (Fig. 3). 
A

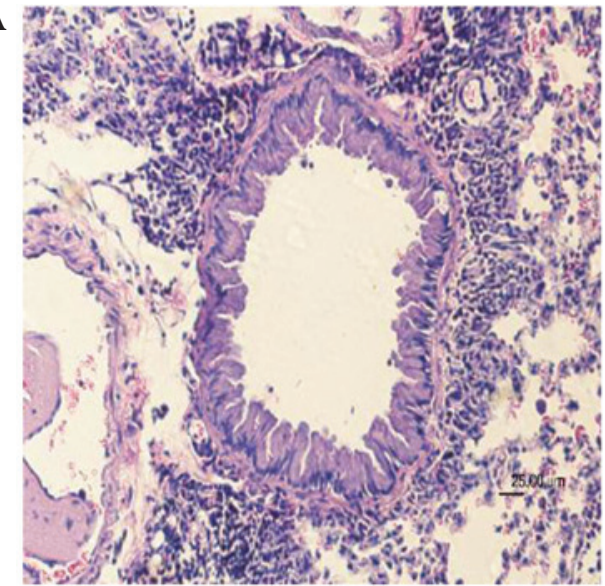

B

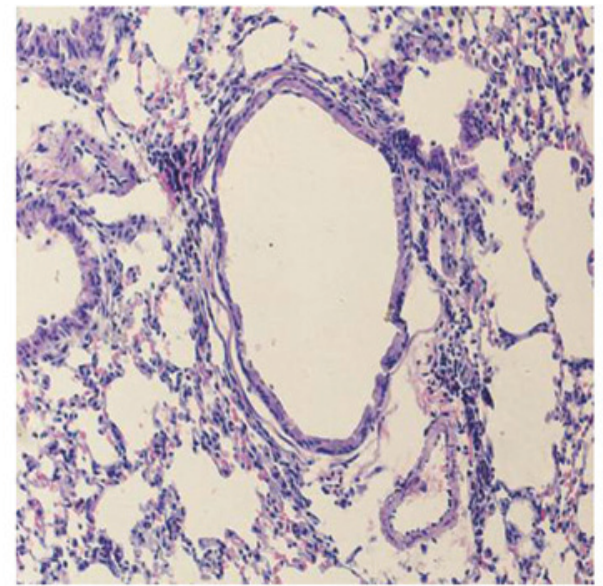

Figure 1. Asthmatic and control group histopathology (hematoxylin and eosin staining; magnification, $\mathrm{x} 200$ ). (A) Pathological sections showed eosinophilic airway inflammation of the mice from the asthmatic group. Lung tissue from the asthmatic mice revealed peribronchiolar and perivascular infiltrates, mainly consisting of eosinophils and lymphocytes, with airway stenosis and inflammatory cells scattered in the lung interstitium. (B) No significant pathologial changes were observed in the control group. The histopathological examination was carried out thrice for each mouse with similar results, and representative images are shown.

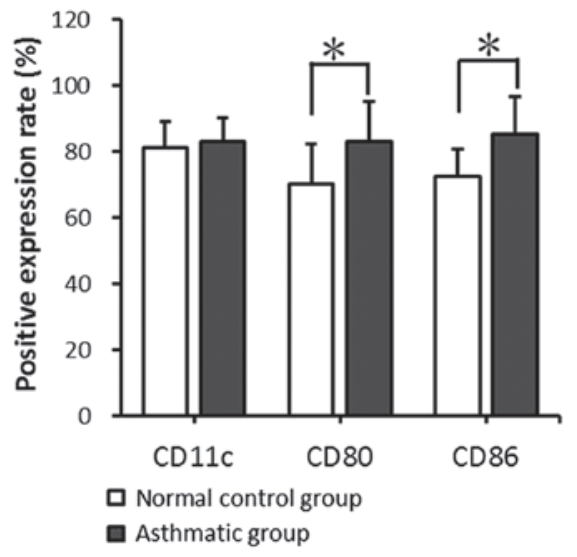

Figure 2. Positive expression rates of CD11c, CD80 and CD86 on mDCs. The positive expression rates of $\mathrm{CD} 11 \mathrm{c}, \mathrm{CD} 80$, and CD86 on mDCs were detected by flow cytometry prior to transfection. In contrast with the normal control group, the asthmatic group showed comparable CD11c expression levels and significantly higher CD80 and CD86 expression levels. "P $<0.05$ vs. the control group. $\mathrm{CD}$, cluster of differentiation; $\mathrm{mDCs}$, mature dendritic cells.

$m R N A$ and protein expression of $C D 80$ and $C D 86$ by $\mathrm{mDCs}$ following transfection. The mRNA and protein expression levels of CD80 and CD86 in the transfected mDCs were detected by RT-qPCR and FACS analysis at 24 and $72 \mathrm{~h}$, respectively, after transfection. The CD80 and CD86 mRNA expression levels detected by RT-qPCR indicated that CD80 mRNA expression levels in the non-siRNA, siRNA and negative siRNA groups were $2.09 \pm 0.46,0.60 \pm 0.17$, and $2.04 \pm 0.93$, respectively, and the CD86 mRNA expression levels were $3.58 \pm 0.20,0.91 \pm 0.48$, and $2.83 \pm 0.83$, respectively. The data provided by FACS indicated that the CD80 protein positive expression rates in the non-siRNA, siRNA and negative siRNA groups were $82.45 \pm 15.80,30.79 \pm 7.07$ and $81.83 \pm 10.07 \%$, respectively, and the CD86 protein positive expression rates were $89.45 \pm 10.22,27.29 \pm 6.99$, and $87.66 \pm 11.74 \%$, respectively. The mRNA expression level and protein positive expression rates exhibited comparable results. There was no significant significance between the
$\mathbf{A}$

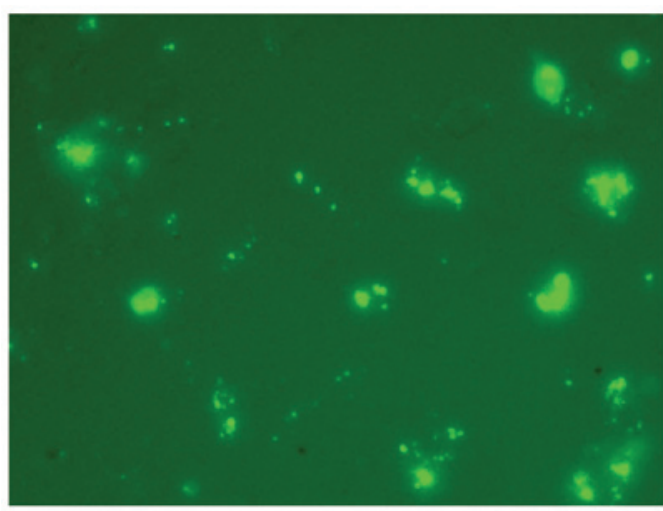

B

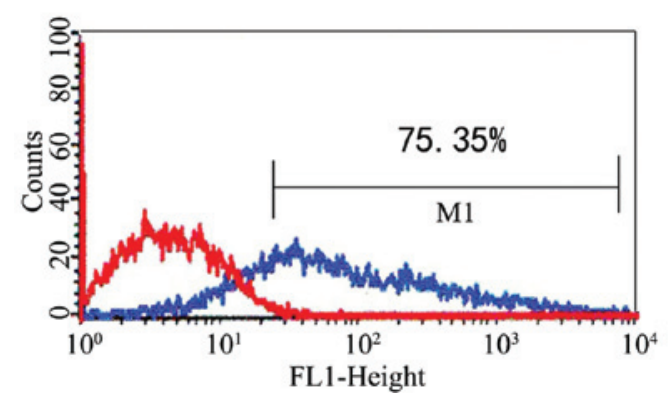

Figure 3. Efficiency of siRNA transfection in mDCs. Transfection efficiency was (A) observed under fluorescence microscope and (B) detected by flow cytometry at $6 \mathrm{~h}$ post-transfection. (A) Through the fluorescence microscope, fluorescent cells transfected with FAM-scrambled-siRNA exhibited green fluorescence. (B) FAM-conjugated siRNA uptake was analyzed by flow cytometry. Histograms represent cell numbers at various fluorescence intensities of isotype control (red) and transfected DCs (blue). siRNA transfection efficiency was $\sim 75 \%$, suggesting that the $\mathrm{mDC}$ had been successfully transfected by specific siRNA and were suitable for use in subsequent experiments. Data are representative of three independent experiments for each group. siRNA, small interfering RNA; mDCs, mature dendritic cells.

non-siRNA group and the negative siRNA group $(\mathrm{P}>0.05)$. CD80 and CD86 expression at the mRNA and protein levels in the siRNA group decreased significantly compared with that in the non-siRNA and negative siRNA groups $(\mathrm{P}<0.05$; 


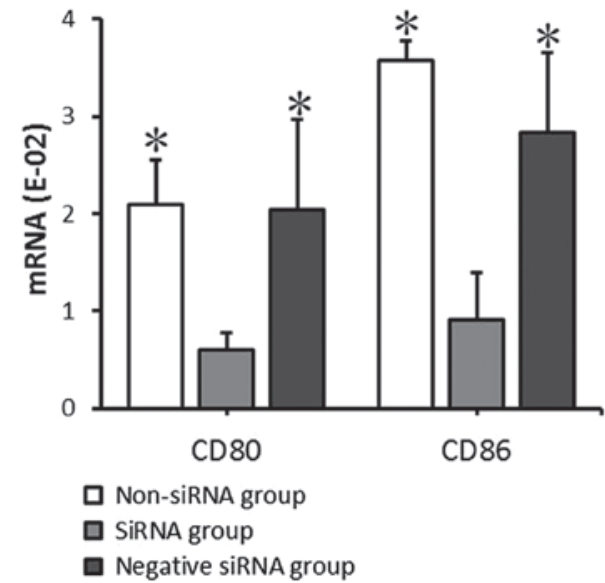

Figure 4. CD80 and CD86 mRNA expression levels detected by reverse transcription-quantitative polymerase chain reaction, performed at $24 \mathrm{~h}$ post-transfection. The CD80 and CD86 mRNA expression levels in the siRNA group (transfected with CD80 and CD80 siRNA) were significantly decreased compared with those in the non-siRNA and negative siRNA groups. No significant significance was found between the non-siRNA and negative siRNA groups. " $\mathrm{P}<0.05$ vs. the siRNA group. $\mathrm{CD}$, cluster of differentiation; siRNA, small interfering RNA.

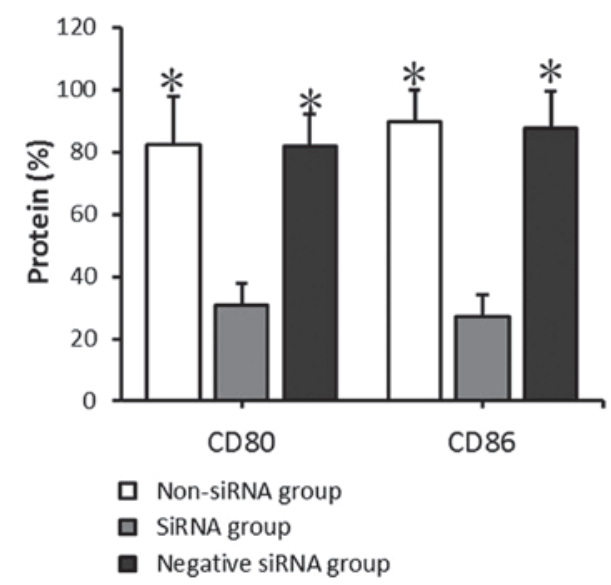

Figure 5. CD80 and CD86 protein positive expression rates detected by fluorescence-activated cell sorting. The CD80 and CD86 protein positive expression rates in the siRNA group (transfected with CD80 and CD80 siRNA) were significantly decreased compared with those in the non-siRNA and negative siRNA groups. No significant significance was found between the non-siRNA and negative siRNA group. " $\mathrm{P}<0.05$ vs. the siRNA group. $\mathrm{CD}$, cluster of differentiation; siRNA, small interfering RNA.

Figs. 4 and 5). This demonstrates that siRNA-targeted interference can significantly suppress CD80 and CD86 mRNA and protein expression levels.

IFN- $\gamma$ and IL-4 secretion by T cells co-cultured with $\mathrm{mDCs}$. After $72 \mathrm{~h}$ of co-culture, IFN- $\gamma$ and IL-4 levels in the supernatant of the $\mathrm{mDC}$ and T-cell co-culture system were detected by ELISA. IFN- $\gamma$ expression was significantly increased in the siRNA group $(132.73 \pm 25.04 \mathrm{pg} / \mathrm{ml})$, as compared with the non-siRNA and negative siRNA groups $(72.56 \pm 26.30$ and $80.21 \pm 24.42 \mathrm{pg} / \mathrm{ml}$, respectively; $\mathrm{P}<0.05$ ), whereas no significant differences were detected between the non-siRNA and negative siRNA groups $(\mathrm{P}>0.05)$. IL-4 expression levels were significantly decreased in the siRNA group $(93.04 \pm 23.13 \mathrm{pg} / \mathrm{ml})$, as

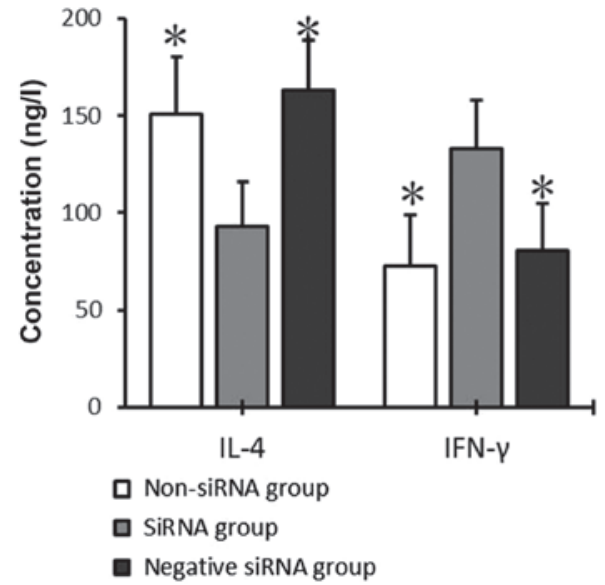

Figure 6. IFN- $\gamma$ and IL-4 levels in the supernatant of an $\mathrm{mDC}$ and $\mathrm{T}$ cell co-culture system as detected by enzyme-linked immunosorbent assay. The IFN- $\gamma$ level in the siRNA group (transfected with CD80 and CD80 siRNA) was significantly increased compared with that in the non-siRNA and negative siRNA groups, while IL-4 decreased significantly. There was no significant difference between the non-siRNA and negative siRNA groups. ${ }^{*} \mathrm{P}<0.05$ vs. the siRNA group. IFN, interferon; IL, interleukin; mDC, mature dendritic cell; siRNA, small interfering RNACD, cluster of differentiation.

compared with the non-siRNA and negative siRNA groups $(150.69 \pm 29.50$ and $163.19 \pm 25.36 \mathrm{pg} / \mathrm{ml}$, respectively; $\mathrm{P}<0.05)$, whereas no significant differences were detected between the non-siRNA and negative siRNA groups ( $\mathrm{P}>0.05$; Fig. 6$)$.

\section{Discussion}

Bronchial asthma is a chronic inflammatory airway disease involving a variety of inflammatory cells, including mast cells, eosinophils, lymphocytes and other cell components (14-17). Th1/Th2 imbalance is a key factor contributing to asthma severity (18). APCs, including DCs, macrophages and B cells (19), play a crucial role in the stimulation of T cells (3). Among those, the DC is the most powerful APC, contributing to primary and secondary immune responses, including allergic immunity. Mature DCs (mDCs) with high levels of expression of the co-stimulatory molecules CD80 and CD86 can activate $\mathrm{T}$ cells, while immature dendritic cells (iDCs) with a low level of expression of CD80 and CD86 suppress the T-cell response and induce immune tolerance $(20,21)$. DCs in patients with asthma have been demonstrated to be hyperactive (22).

CD80 and CD86 are two types of protein that are expressed on the APC surface, and which work in tandem to provide co-stimulatory signals necessary for T-cell activation and survival. Previous studies have shown that the expression levels of the co-stimulatory molecules CD80 and CD86 on the $\mathrm{mDC}$ surface are closely associated with Th2-cell reaction and airway inflammation $(23,24)$. In asthmatic patients, mDCs that highly express CD80 and CD86 can stimulate naïve $\mathrm{CD} 4^{+}$helper T-cell activation to differentiate toward Th2 cells, resulting in a Th1/Th2 imbalance. Following that, the inadequate secretion of Th1 cytokines such as IFN- $\gamma$, along with the increased secretion of Th2 cytokines such as IL-4 and IL-5, causes eosinophilic inflammation and allergic airway inflammation $(10,24,25)$. Two signals are required for 
the promotion of Th2-cell activation (26-28). The first signal is the formation of antigen-MHC complexes on the $\mathrm{mDC}$ surface that bind specifically with the T-cell receptor-CD3 receptor complex on T-cell surfaces, and the second signal is co-stimulatory molecule expression and functional activation on the mDC surfaces that specifically bind to receptors on naïve T cells; the two signals form a co-stimulatory pathway (29). It has also been suggested that there is a third signal (30), as certain cellular molecules produced by DCs, such as thymic stromal lymphopoietin (TSLP), affect the direction of Th-cell differentiation. However, among all the aforementioned signals, the CD80/CD86-CD28 co-stimulatory pathway is the most classic and important. Previous research has indicated that the CD80/CD86-CD28 co-stimulatory pathway may be an effective target for asthma treatment by demonstrating that blocking the CD80/CD86 co-stimulatory pathway by monoclonal antibody approaches can inhibit inflammation in asthmatic mice (25). In addition, suppressing the CD80/CD86 co-stimulatory pathway using antisense oligonucleotides can suppress airway hyperactivity (31).

RNAi is a gene-silencing phenomenon whereby endogenous- or exogenous-specific double-stranded RNAs trigger the degradation of homologous mRNA and induce the loss of corresponding functional phenotypes. Since the technique was first discovered in 1998 by Fire et al (32), the technique has undergone further development to attain a high degree of specificity and efficiency. The therapeutic application of RNAi technology is a topic that has been attracting high levels of interest in basic medical and clinical research in recent years. The ability of RNAi to inhibit virulent gene expression has been widely used to treat a variety of diseases (33-35). Also, a number of studies have reported that RNAi can be used in DCs to diagnose and treat bronchial asthma (36-39). Darcan-Nicolaisen et al (40) discovered that the two major signs of allergic asthma in the OVA-induced asthma mouse model, which are airway inflammation and hyperactivity, were significantly ameliorated by signal transducer and activator of transcription 6 (STAT6) silencing in airway epithelial cells using the administration of siRNA nose drops. Moriwaki et al (41) demonstrated that siRNA-mediated suppressor of cytokine signaling 3 (SOCS3) gene silencing could suppress the airway reactivity and eosinophilic infiltration that was induced by allergenic stimulation in asthmatic mice. Zheng et al (42) found that the application of siRNA was able to inhibit tyrosine protein kinase (TPK) gene expression in the DCs of asthmatic mice, repressing the functional capability of DCs as antigen-presenting cells, thereby inhibiting T-cell activation and differentiation. However, studies concerning the effects of siRNA-mediated CD80 and CD86 knockdown in DCs on T-cell differentiation in asthmatic mice are lacking. In the present study, CD80 and CD86 mRNA and protein expression in murine bone marrow-derived DCs was successfully decreased with CD80- and CD86-targeting siRNA, which verified the efficiency of RNAi.

In the present study, an asthmatic mouse model was used that was established according to previously reported methods (43). The results indicated that the $\mathrm{mDC}$ s obtained from the asthmatic group exhibited increased CD80 and CD86 expression levels, which implies that the CD80/CD86 capacity may be heightened in asthmatic patients. Following transfection of the mDCs with CD80- and CD86-targeted siRNA, the mRNA expression levels and protein positive expression rates of CD80 and CD86 were significantly decreased, confirming the inhibitory effect that the siRNA approach had on the co-stimulatory molecules CD80 and CD86 at the transcriptional and translational levels. In the supernatant from the co-culture of mDCs and $\mathrm{T}$ cells, RNAi induced an increase in IFN- $\gamma$ expression and a reduction of IL-4 levels, indicating that decreasing the expression of the co-stimulatory molecules CD80 and CD86 in mDCs weakened the CD80/CD86-CD28 co-stimulatory pathway in asthmatic mice. RNAi also affected the expression of Th1/Th2 cytokines, indicating that the original Th1/Th2 imbalance was changed and, consequently, immune tolerance was induced. These findings indicate that CD80 and CD86 may be potential targets for RNAi application in asthma treatment, and provide a new avenue for the gene therapy of asthma.

\section{Acknowledgements}

This study was supported by an Open Projects Grants from the State Key Laboratory of Respiratory Disease (Guangzhou, China) (grant no. 2007DA80154F1107).

\section{References}

1. Locksley RM: Asthma and allergic inflammation. Cell 140: 777-783, 2010.

2. Holgate ST: Innate and adaptive immune responses in asthma. Nat Med 18: 673-683, 2012

3. Larché M, Robinson DS and Kay AB: The role of T lymphocytes in the pathogenesis of asthma. J Allergy Clin Immunol 111: 450-464, 2003.

4. Lambrecht BN and Hammad H: The role of dendritic and epithelial cells as master regulators of allergic airway inflammation. Lancet 376: 835-843, 2010

5. Holt PG and Upham JW: The role of dendritic cells in asthma. Curr Opin Allergy Clin Immunol 4: 39-44, 2004.

6. Lim TS, Goh JK, Mortellaro A, Lim CT, Hämmerling GJ and Ricciardi-Castagnoli P: CD80 and CD86 differentially regulate mechanical interactions of T-cells with antigen-presenting dendritic cells and B-cells. PLoS One 7: e45185, 2012.

7. Wong CK, Lun SW, Ko FW, Ip WK, Hui DS and Lam CW: Increased expression of plasma and cell surface co-stimulatory molecules CTLA-4, CD28 and CD86 in adult patients with allergic asthma. Clin Exp Immunol 141: 122-129, 2005.

8. Shi HZ, Xie ZF, Deng JM, Chen YQ and Xiao CQ: Soluble CD86 protein in serum samples of patients with asthma. Thorax 59: 870-875. 2004.

9. Van Rijt LS and Lambrecht BN: Dendritic cells in asthma: a function beyond sensitization. Clin Exp Allergy 35: 1125-34, 2005.

10. Van RijtLS, Vos N, Willart M,Kleinjan A, Coyle AJ,Hoogsteden HC and Lambrecht BN: Essential role of dendritic cell CD80/CD86 costimulation in the induction, but not reactivation, of $\mathrm{TH} 2$ effector responses in a mouse model of asthma. J Allergy Clin Immunol 114: 166-173, 2004.

11. Wu SG, Wang GL, Li LY and Ji J: Effects of microRNA-21 on the interleukin 12/signal transducer and activator of transcription 4 signaling pathway in asthmatic mice. Cent Eur J Immunol 39: 40-45, 2014.

12. Gu X, Xiang J, Yao Y and Chen Z: Effects of RNA interference on CD80 and CD86 expression in bone marrow-derived murine dendritic cells. Scand J Immunol 64: 588-594, 2006.

13. Livak KJ and Schmittgen TD: Analysis of relative gene expression data using real-time quantitative PCR and the $2^{-\Delta \Delta \mathrm{Ct}}$ method. Methods 25: 402-408, 2001.

14. Fanta CH: Asthma. N Engl J Med 360: 1002-1014, 2009.

15. Lambrecht $\mathrm{BN}$ and Hammad $\mathrm{H}$ : Lung dendritic cells in respiratory viral infection and asthma: From protection to immunopathology. Annu Rev Immunol 30: 243-270, 2012.

16. Wenzel SE: Asthma phenotypes: The evolution from clinical to molecular approaches. Nat Med 18: 716-725, 2012.

17. Hansel TT, Johnston SL and Openshaw PJ: Microbes and mucosal immune responses in asthma. Lancet 381: 861-873, 2013 
18. Compalati E, Braido F and Canonica GW. An update on allergen immunotherapy and asthma. Curr Opin Pulm Med 20: 109-117, 2014.

19. Goyvaerts C, Dingemans J, De Groeve K, Heirman C, Van Gulck E, Vanham G, De Baetselier P, Thielemans K, Raes G and Breckpot $\mathrm{K}$ : Targeting of human antigen-presenting cell subsets. J Virol 87: 11304-11308, 2013.

20. Reis e Sousa C: Dendritic cells in a mature age. Nat Rev Immunol 6: 476-483, 2006.

21. Gill MA: The role of dendritic cells in asthma. J Allergy Clin Immunol 129: 889-901, 2012.

22. Shi JH, LI YG and LI TS: The roles of dendritic cells in antigen presentation and the pathogenesis of asthma. Zhonghua Jie He He Hu Xi Za Zhi 28: 22-27, 2005 (In Chinese).

23. Wong CK, Lun SW, Ko FW, Ip WK, Hui DS and Lam CW: Increased expression of plasma and cell surface co-stimulatory molecules CTLA-4, CD28 and CD86 in adult patients with allergic asthma. Clin Exp Immunol 141: 122-129, 2005.

24. Bellou A and Finn PW: Costimulation: Critical pathways in the immunologic regulation of asthma. Curr Allergy Asthma Rep 5: 149-154, 2005.

25. Chen YQ and Shi HZ: CD28/CTLA-4-CD80/CD86 and ICOS-B7RP-1 costimulatory pathway in bronchial asthma. Allergy 61: 15-26, 2006.

26. Bieber T, Novak N, Herrmann N and Koch S: Role of dendritic cells in atopic dermatitis: An update. Clin Rev Allergy Immunol 41: 254-258, 2011.

27. Hespel C and Moser M: Role of inflammatory dendritic cells in innate and adaptive immunity. Eur J Immunol 42 : 2535-2543, 2012.

28. Novak N: An update on the role of human dendritic cells in patients with atopic dermatitis. J Allergy Clin Immunol 129: 879-886, 2012.

29. Lombardi V, Singh AK and Akbari O: The role of costimulatory molecules in allergic disease and asthma. Int Arch Allergy Immunol 151: 179-189, 2010.

30. Zhang Y, Zhou X and Zhou B: DC-derived TSLP promotes Th2 polarization in LPS-primed allergic airway inflammation. Eur J Immunol 42: 1735-1743, 2012.

31. Crosby JR, Guha M, Tung D, Miller DA, Bender B Condon TP, York-DeFalco C, Geary RS, Monia BP, Karras JG and Gregory SA: Inhaled CD86 antisense oligonucleotide suppresses pulmonary inflammation and airway hyper-responsiveness in allergic mice. J Pharmacol Exp Ther 321: 938-946, 2007.
32. Fire A, Xu S,Montgomery MK, Kostas SA, Driver SE and Mello CC: Potent and specific genetic interference by double-stranded RNA in Caenorhabditis elegans. Nature 391: 806-811, 1998.

33. Ghafouri-Fard S: siRNA and cancer immunotherapy. Immunotherapy 4: 907-917, 2012.

34. Gavrilov K and Saltzman WM: Therapeutic siRNA: Principles, challenges and strategies. Yale J Biol Med 85: 187-200, 2012.

35. Yan WJ, Xu SJ, Xie MM and Wu BL: Effects of exogenous cyclic dimeric guanosinemonophosphate on the gene expression of Streptococcus mutans. Zhongguo Zu Zhi Gong Cheng Yan Jiu 16: 1451-1454, 2012 (In Chinese).

36. Lee CC, Huang HY and Chiang BL: Lentiviral-mediated interleukin-4 and interleukin-13 RNA interference decrease airway inflammation and hyperresponsiveness. Hum Gene Ther 22: 577-686, 2011.

37. Li Y, Sun M, Cheng H, Li S, Liu L, Qiao H, Hua S and Lu J: Silencing IL-23 expression by a small hairpin RNA protects against asthma in mice. Exp Mol Med 43: 197-204, 2011.

38. Woska JJ and Gillespie ME: Small-interfering RNA-mediated identification and regulation of the ternary SNARE complex mediating RBL-2H3 mast cell degranulation. Scand J Immunol 73: 8-17, 2011.

39. Lu XX, McCoy KS, Xu JL, Hu WK and Chen HB: Small interfering RNA targeting T-cell Ig mucin-3 decreases allergic airway inflammation and hyperresponsiveness. Inflammation 36: 582-591, 2013.

40. Darcan-Nicolaisen Y, Meinicke H, Fels G, Hegend O, Haberland A, Kühl A, Loddenkemper C, Witzenrath M, Kube S, Henke W and Hamelmann E: Small interfering RNA against transcription factor STAT6 inhibits allergic airway inflammation and hyperreactivity in mice. J Immunol 182: 7501-7508, 2009.

41. Moriwaki A, Inoue H, Nakano T, Matsunaga Y, Matsuno Y, Matsumoto T, Fukuyama S, Kan-O K, Matsumoto K, Tsuda-Eguchi M, et al: T cell treatment with small interfering RNA for suppressor of cytokine signaling 3 modulates allergic airway responses in a murine model of asthma. Am J Respir Cell Mol Biol 44: 448-455, 2011.

42. Zheng YH, Lu JJ, Guo ZL, Ren T and Liang YJ: Small interfering RNAs specific for spleen tyrosine kinase inhibit maturation of dendritic cells of asthmatic mice in vitro. Zhongguo Hu Xi Yu Wei Zhong Jian Hu Za Zhi. 8: 487-491, 2009 (In Chinese).

43. Li JG, Zhuansun YX, Ran PX, Zhang W, Mo XN, Wu H and Li YQ: Effects of bone marrow mesenchymal stem cells on $\mathrm{CD} 4^{+} \mathrm{CD} 25^{+}$ regulatory $\mathrm{T}$ cells and airway inflammation in asthmatic mice. Zhongguo Zu Zhi Gong Cheng Yan Jiu 12: 9302-9305, 2008 (In Chinese). 